\title{
Isobutane Alkylation Process Synthesis by means of Hybrid Simulation-Multiobjective Optimization
}

\author{
María J. Fernández-Torres*, Norberto García, José A. Caballero \\ Department of Chemical Engineering,University of Alicante, Apdo 99 E-03080, \\ Alicante, Spain \\ fernandez@ua.es
}

\begin{abstract}
Multiobjective Generalized Disjunctive Programming (MO-GDP) optimization has been used for the synthesis of an important industrial process, isobutane alkylation. The two objective functions to be simultaneously optimized are the environmental impact, determined by means of LCA (Life Cycle Assessment), and the economic potential of the process. The main reason for including the minimization of the environmental impact in the optimization process is the widespread environmental concern by the general public. For the resolution of the problem we employed a hybrid simulationoptimization methodology, i.e., the superstructure of the process was developed directly in a chemical process simulator connected to a state of the art optimizer. The model was formulated as a GDP and solved using a logic algorithm that avoids the reformulation as MINLP -Mixed Integer Non Linear Programming-. Our research gave us Pareto curves compounded by three different configurations where the LCA has been assessed by two different parameters: global warming potential and ecoindicator-99.
\end{abstract}

Keywords: Generalized Disjunctive Programming (GDP), Multiobjective (MO) optimization, Isobutane alkylation, Life Cycle Assessment (LCA).

\section{Introduction}

Environmental considerations have only been recently included into process engineering optimization. Traditionally any process optimization was based on profitability. The best way to include the environmental criteria into the process design is by multiobjective (MO) optimization (Azapagic 1999). Life cycle assessment (LCA) provides a comprehensive means to account for environmental impact as indicated by the same author. Since then many examples of MO optimization can be found in literature, for instance, (Ruiz-Femenia et al. 2013) made use of this methodology to optimize chemical supply chains. (Wang et al. 2013) employed MO optimization to the configuration of a hydrocarbon biorefinery. (Gebreslassie et al. 2013a) obtained the sustainable design of a hydrocarbon biorefinery via fast pyrolysis. (Gebreslassie et al. $2013 \mathrm{~b}$ ) presented the synthesis of an algae-based biorefinery that includes carbon sequestration.

In this paper we present the MO optimization methodology applied to the isobutane alkylation process. Our aim is to show how the inclusion of LCA into the MO optimization is a helpful procedure for industrial process design and optimization. As a 
result, we obtain a set of flowsheet configurations (together with its process variables) able to manufacture isooctane that simultaneously optimizes the production cost and minimizes the associated environmental damage.

\section{Procedure for the synthesis of the optimized process}

Three steps are necessary for the synthesis of the optimized isobutane alkylation process:

- First step is to propose a superstructure of alternatives. Ours (Figure 1) is based on the licensed processes by Stratford Engineering Corporation and Exxon Research and Engineering. To that base we added extra alternatives that include: an extension of the post reaction separation alternatives, the possibility of feed purification as pretreatment, existence of purge, etc. Two main reactions have been considered for the present study: $\mathrm{C} 4+\mathrm{i}-\mathrm{C} 4 \rightarrow \mathrm{C} 8$ (main reaction) and $\mathrm{C} 4+\mathrm{C} 8 \rightarrow \mathrm{C} 12$ (unwanted reaction) where $\mathrm{C} 4$ stands for 1-butene, $\mathrm{i}-\mathrm{C} 4$ for isobutane, $\mathrm{C} 8$ for isooctane and $\mathrm{C} 12$ for dodecane. Propane (C3) inevitably enters with 1-butene to the process but behaves like an inert substance. Presence of sulfuric acid is required in the reactor chamber to act as a catalyst. This acid is later separated by means of a settler and recirculated back to the reactor.

- Second step is to write the mathematical model as a hybrid simulation-optimization multi-objective generalized disjunctive programming (GDP) problem (see section 3).

- Third step is to solve the GDP problem using logic-based algorithms without reformulation to MINLP (Türkay et al. 1996) (see section 3).

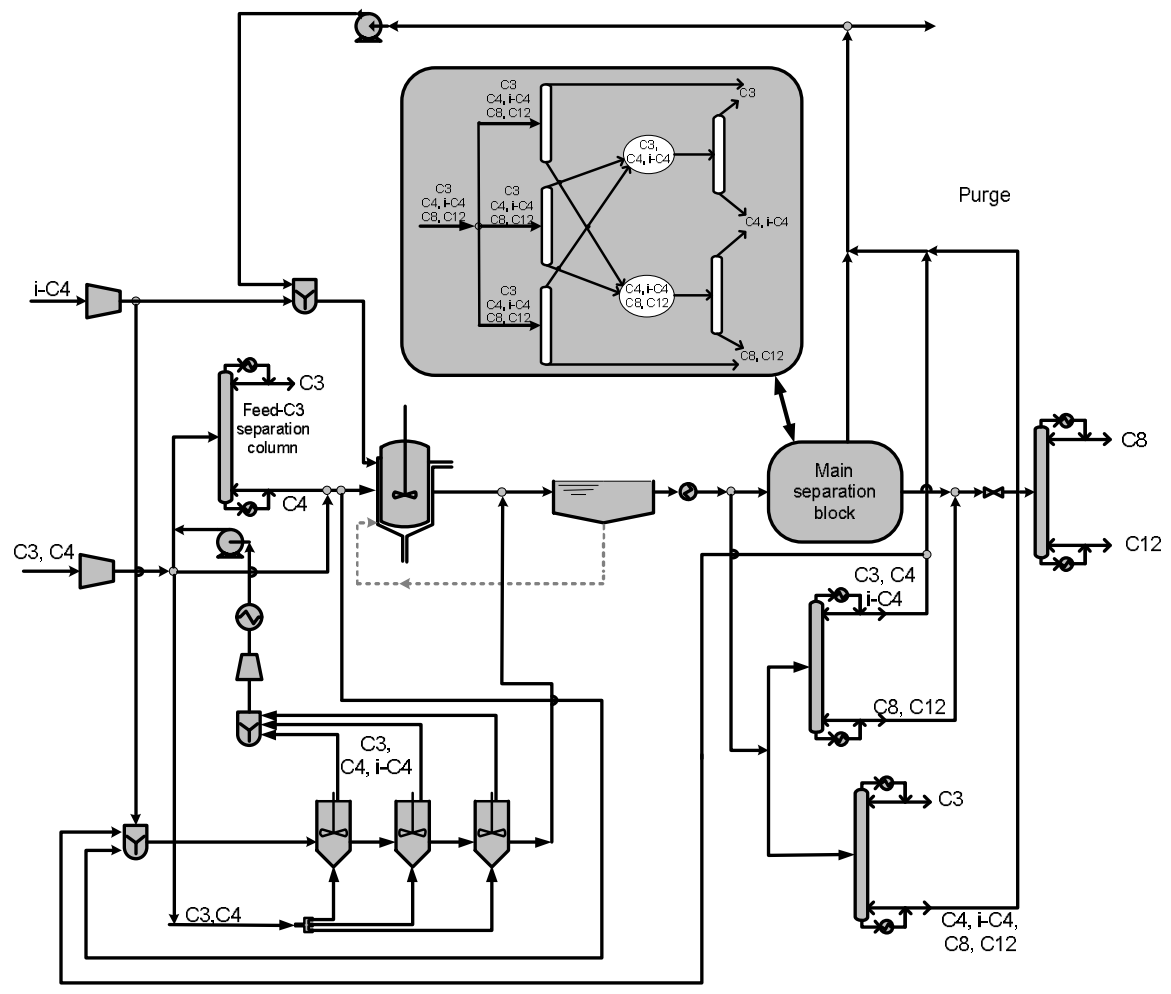

Figure 1. Superstructure considered in the present study. 
Isobutane Alkylation Process Synthesis by means of Hybrid SimulationMultiobjective Optimization

\section{Mathematical model}

Conceptually the model can be written as follows:

$$
\begin{aligned}
& \min :\left\{f_{E c}\left(\mathbf{x}_{\mathbf{D}}, \mathbf{x}_{\mathbf{I}}\right), f_{E n}\left(\mathbf{x}_{\mathbf{D}}, \mathbf{x}_{\mathbf{I}}\right)\right\} \\
& \text { s.t. } \quad \mathbf{x}_{\mathbf{D}}=\mathbf{r}_{i m}\left(\mathbf{x}_{\mathbf{I}}\right) \\
& \mathbf{r}_{e x}\left(\mathbf{x}_{\mathbf{D}}, \mathbf{x}_{\mathbf{I}}\right)=\mathbf{0} \\
& \mathbf{s}_{e x}\left(\mathbf{x}_{\mathbf{D}}, \mathbf{x}_{\mathbf{I}}\right) \leq \mathbf{0} \\
& {\left[\begin{array}{l}
Y_{i} \\
\mathbf{x}_{\mathbf{D}}=\mathbf{h}_{i m, i}\left(\mathbf{x}_{\mathbf{I}, i}\right) \\
\mathbf{h}_{e x, i}\left(\mathbf{x}_{\mathbf{D}}, \mathbf{x}_{\mathbf{I}, i}\right)=\mathbf{0} \\
\mathbf{g}_{e x, i}\left(\mathbf{x}_{\mathbf{D}}, \mathbf{x}_{\mathbf{I}, i}\right) \leq \mathbf{0}
\end{array}\right] \underline{\vee}\left[\begin{array}{c}
\neg Y_{i} \\
\mathbf{x}_{\mathbf{I}, i}=\mathbf{0}
\end{array}\right] \forall i \in D} \\
& \Omega(\mathbf{Y})=\operatorname{True} \\
& \mathbf{x}_{\mathbf{I} \in X \subseteq \Re^{\mathrm{n}}}
\end{aligned}
$$

Where $f_{E c}$ and $f_{E n}$ are the economic and the environmental objective functions respectively. $\mathbf{x}_{\mathbf{D}}$ is a vector of dependent variables like those calculated by the process simulator used (Aspen-Hysys ${ }^{\mathrm{TM}}$ ). $\mathbf{x}_{\mathbf{I}}$ is a vector of independent variables on which the programmer has full control. The index "im" stands for implicit equation calculated by third party model (process simulator, other matlab models, etc.). The index "ex" indicates explicit equation. The boolean variable $Y_{i}$ takes only two values, true if equipment " $i$ " is selected or false if not. Finally $p$ denotes the number of Boolean variables.

Eq. (5) is a disjunction that explicitly indicates that if a given item of equipment is selected, then all the equations related to it must be used, if not, all the independent variables corresponding to that item of equipment are set to zero. This equation only allow for two term disjunction which is the procedure for process network and synthesis. The equations related to each equipment are the sizing, costing and environmental impact.

All the thermodynamic data required and all of the equipment simulation (excluding those corresponding to the distillation columns) are calculated by Aspen-Hysys ${ }^{\mathrm{TM}}$. The distillation columns simulations and sequence optimization were performed according to the procedure described in (Caballero et al. 2001), (Caballero et al. 2006) and (Caballero et al. 2013). The simulation of thermal couples is performed by the methodology described in (Navarro-Amorós et al. 2013). 
Eq. (6) of the model represents all the logical relationships used with the intention to obtain feasible configurations. This collection of relationships includes:

- Select the isothermal or the three autorefrigerated reactors.

- The inert substance (C3) is either removed before entering the system with a prefractionator or at a later stage in the main separation block shown in Figure 1.

- At most only one of the initial separations in the main separation block can be selected after the reaction. Such a statement must be necessarily followed by a connectivity relation to indicate which separation or separations can follow.

- Finally, another set of logical relationships are necessary to indicate if reboilers and/or condensers are to be used in the different distillation columns. This is necessary since we are explicitly including thermally coupled distillation alternatives.

Once the mathematical model to describe the MO optimization problem is completed we proceed to its resolution. To achieve that we used the epsilon constraint method (Ehrgott 2005). The results are obtained as Pareto curves (environmental indicators vs economic potential), the steps are the following:

- First step, find the two extremes of the Pareto curves by optimizing each individual objective (single-objective optimization).

- Second step, reformulate the model (equations 1 to 8 ) by moving the environmental objective equation to the constraints.

- Third step, solve a sequence of problems with a single objective by changing the value of epsilon (Ehrgott 2005) between the two extremes of the Pareto curve found on the first step.

This type of problems is quite complex, therefore it is convenient to perform a sensitivity analysis beforehand to decide which variables are convenient to use as independent variables. Our study indicated that we should employ the following: the molar flowrate ratio of the two fresh input streams to the system, the volume of the reactor(s), the temperature of the isothermal reactor, the split fraction in the purge stream and the individual flows and temperature in the recycle stream.

Some parameter values that have been fixed are: the recovery of the key component in the distillation column (0.99) and the reflux ratio in the distillation column (1.3).

\section{Results}

As indicated in the previous section, the results have been obtained as Pareto curves (see Fig. 2) where the environmental impact is plotted against the economic potential. Each curve corresponds to the MO optimization process conducted with a different environmental indicators, global warming potential (GWP) and ecoindicator-99 (Ei-99). It can be seen in the figure that each curve is compounded by three distinctive sections which correspond to three different processes also shown and depicted by A, B and C. 
Isobutane Alkylation Process Synthesis by means of Hybrid Simulation-
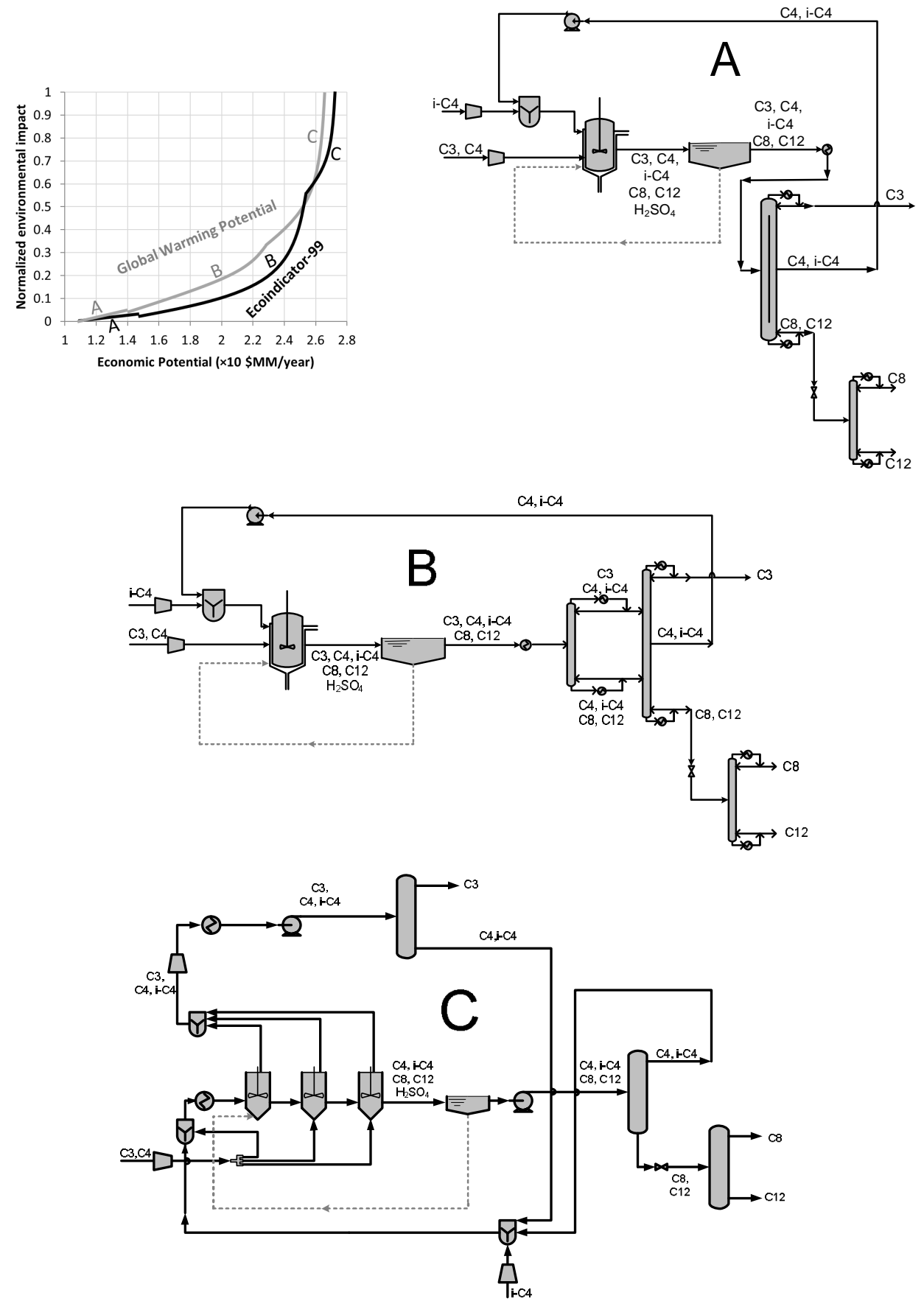

Figure 2. Comparison between the Pareto's curves obtained using both environmental indicators (GWP and Ei-99) together with the three structures that compound them (A, B and C). A is a configuration where the main separation is achieved with a divided wall column. B is a configuration where the separation uses a prefractionator without thermal couple. And $\mathrm{C}$ is a configuration where the reaction takes place in three auto-refrigerated reactors in series. 


\section{Conclusions}

An important industrial process (isobutene alkylation) is synthesized by means of hybrid simulation multiobjective optimization. Each of the Pareto curves we obtained refers to three different structures. The designer(s) can now decide at which point of the optimized solution they would like to work by choosing any point on these curves. Our suggestion would be to choose a point on line segment $C$ in Figure 2 because of its steep slope toward the right end of the curve (maximum economic potential). In other words, we could like to choose a point to the left to that which maximizes only the economic potential, but still on the steep part of the curve, in order to obtain substantially less environmental impact without significantly affecting the economic gains.

The present study shows that GDP is the ideal framework to solve hybrid problems of simulation-optimization with the final aim to perform successful process synthesis.

Finally, we believe that the solution encountered and presented here, is likely to be the global optimal solution since the Pareto curves obtained show smooth shapes and they are reached from different starting points.

\section{Acknowledgement}

We acknowledge the support from the Spanish Ministry of Science and Innovation (CTQ2012-37039-C02-02)

\section{References}

Azapagic, A., 1999, Life cycle assessment and its application to process selection, design and optimisation. Chemical Engineering Journal, 73,1: 1-21.

Caballero, J. A. and I. E. Grossmann, 2001, Generalized Disjunctive Programming Model for the Optimal Synthesis of Thermally Linked Distillation Columns. Industrial \& Engineering Chemistry Research, 40,10: 2260-2274.

Caballero, J. A. and I. E. Grossmann, 2006, Structural Considerations and Modeling in the Synthesis of Heat-Integrated-Thermally Coupled Distillation Sequences. Industrial \& Engineering Chemistry Research, 45,25: 8454-8474.

Caballero, J. A. and I. E. Grossmann, 2013, Synthesis of complex thermally coupled distillation systems including divided wall columns. AIChE Journal, 59,4: 1139-1159.

Ehrgott, M., 2005. Multicriteria optimization. Berlin ; New York, Springer.

Gebreslassie, B. H., M. Slivinsky, et al., 2013a, Life cycle optimization for sustainable design and operations of hydrocarbon biorefinery via fast pyrolysis, hydrotreating and hydrocracking. Computers and Chemical Engineering, 50: 71-91.

Gebreslassie, B. H., R. Waymire, et al., 2013b, Sustainable design and synthesis of algae-based biorefinery for simultaneous hydrocarbon biofuel production and carbon sequestration. AIChE Journal, 59,5: 1599-1621.

Navarro-Amorós, M. A., J. A. Caballero, et al., 2013, An alternative disjunctive optimization model for heat integration with variable temperatures. Computers \& Chemical Engineering, 56,0: 12-26.

Ruiz-Femenia, R., G. Guillén-Gosálbez, et al., 2013, Multi-objective optimization of environmentally conscious chemical supply chains under demand uncertainty. Chemical Engineering Science, 95: 1-11.

Türkay, M. and I. E. Grossmann, 1996, Logic-based MINLP algorithms for the optimal synthesis of process networks. Computers \& Chemical Engineering, 20,8: 959-978.

Wang, B., B. H. Gebreslassie, et al., 2013, Sustainable design and synthesis of hydrocarbon biorefinery via gasification pathway: Integrated life cycle assessment and technoeconomic analysis with multiobjective superstructure optimization. Computers and Chemical Engineering, 52: 55-76. 